\title{
Dielectric Relaxation in Hydrogen Bonded Urea-Based Supramolecular Polymer $N, N^{\prime}$-di(2,2-dipentylheptyl)urea
}

\author{
J. JADŻYN ${ }^{a}$, L. BouteILleR ${ }^{b}$, J.-L. DÉJARDiN ${ }^{c}$ \\ AND G. Czechowski ${ }^{a}$ \\ ${ }^{a}$ Institute of Molecular Physics, Polish Academy of Sciences \\ M. Smoluchowskiego 17, 60-179 Poznań, Poland \\ ${ }^{b}$ Laboratoire de Chimie des Polymères, UMR 7610 \\ Université Pierre et Marie Curie \\ 4 place Jussieu, 75252 Paris Cedex 05, France \\ ${ }^{c}$ Groupe de Physique Statistique et Moléculaire, MEPS
}

Université de Perpignan, 52 Avenue Paul Alduy, 66860 Perpignan Cedex, France

(Received May 29, 2006)

\begin{abstract}
Results of the dielectric relaxation studies, performed for supramolecular polymer formed by $N, N^{\prime}$-di(2,2-dipentylheptyl)urea dissolved in carbon tetrachloride, are presented. The measurements were done for $N, N^{\prime}$ -di(2,2-dipentylheptyl)urea concentration up to about $7 \%$ (in mole fraction) in the frequency region from $100 \mathrm{kHz}$ to $100 \mathrm{MHz}$ and at the temperatures from $5^{\circ} \mathrm{C}$ to $50^{\circ} \mathrm{C}$. The analysis of the experimental data were performed with the Havriliak-Negami equation. In the studied range of $N, N^{\prime}-\operatorname{di}(2,2-$ -dipentylheptyl)urea concentration and temperature, the obtained values of exponents $\alpha$ and $\beta$ of the Havriliak-Negami equation are equal to $0.9 \pm 0.1$ and $0.7 \pm 0.1$, respectively, showing an anomaly in the dielectric relaxation behavior close to the Davidson-Cole type. Two examples of the modeling of dielectric properties of the supramolecular polymer solutions were presented.

PACS numbers: 61.82.Pv, 77.22.Gm, 81.16.Fg, 82.30.Rs
\end{abstract}

\section{Introduction}

Supramolecular polymers are a new class of compounds the synthesis of which have been strongly developed in the last decade. They are forming in a spontaneous assembling process of self-complementary single molecules 
(monomers) through directional, non-covalent bonds created between them (mostly the hydrogen bonds) [1-4]. So, the supramolecular polymers form the most recent branch of "chemistry beyond the covalent bond" [1]. Due to a relatively low energy of the hydrogen bonds (typically $25 \mathrm{~kJ} / \mathrm{mol}$ ), such type of linkage between the basic elements of supramolecular polymers is reversible, in the meaning that it can be much more easily destroyed by the thermal energy $(\approx 0.5 \mathrm{~kJ} / \mathrm{mol})$ as compared to covalent linkages in the conventional polymers (typically $500 \mathrm{~kJ} / \mathrm{mol}$ ). Ecological significance of that property is evident. In general, intermolecular reversible linkage of the hydrogen bonds type, leads to numerous unusual properties of supramolecular polymers in comparison to those of the conventional ones. First of all, the reversible bonds allow one to control the basic property of the polymers - the mean degree of polymerization. It can be simply done by changing the temperature or concentration of self-assembling compound dissolved in an appropriate solvent. So, one gains a possibility to engineer the multi-molecular system of a given, well-defined physical property. Two examples of such type of engineering are presented in this paper.

Among a great variety of compounds which are able to form supramolecular polymers in solutions, $N, N^{\prime}$-dialkylureas (R-NH.CO.NH-R) take a special place [5-12]. Three active centers of the ureide group, $-\mathrm{NH} . \mathrm{CO} . \mathrm{NH}-$, capable of forming the hydrogen bonds $\mathrm{C}=\mathrm{O} \ldots \mathrm{H}-\mathrm{N}$, are essential in the determination of the properties and activity of dialkylureas in all media [13-15]. In 1987 we have proposed [16] the model of the structure of a dialkylurea linear aggregate, in which one molecule of urea is involved with four hydrogen bonds (similar to the hydrogen-bonded water molecules), as shown in Fig. 1. Such type of molecular self-assembling allowed one to explain an unusually strong increase in the molec-

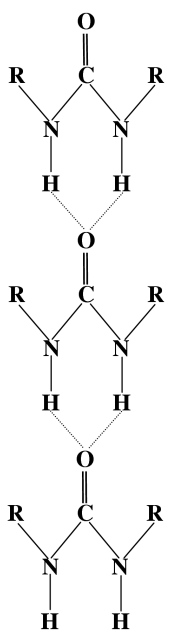

Fig. 1. Model of the linear self-assembled $N, N^{\prime}$-dialkylurea molecules linked with four $\mathrm{C}=\mathrm{O} \ldots \mathrm{H}-\mathrm{N}$ hydrogen-bonds [16]. 
ular apparent dipole moment, observed with an increase in the concentration of dialkylurea in non-polar medium. The model played an important role in understanding the exceptional ability to molecular self-association of a large family of urea-containing supramolecular polymers and organogelators.

In view of the type of interactions linking the molecular components together, the supramolecular chains have a dynamical character. So, the dielectric relaxation studies can provide here an important piece of information on the molecular dynamics through watching the ways of equilibration of the dipolar system after its perturbation by an external electric field. The present paper contains the dielectric relaxation data on the solutions of supramolecular polymer formed by the molecules of $N, N^{\prime}$-dialkylurea with relatively large substituents $\mathrm{R}$.

\section{Experimental}

The supramolecular polymer studied is $N, N^{\prime}$-di(2,2-dipentylheptyl)urea (DPHU) (Fig. 2), dissolved in the non-polar medium (carbon tetrachloride). Synthesis and purification of DPHU were described previously [17]. Carbon tetra-

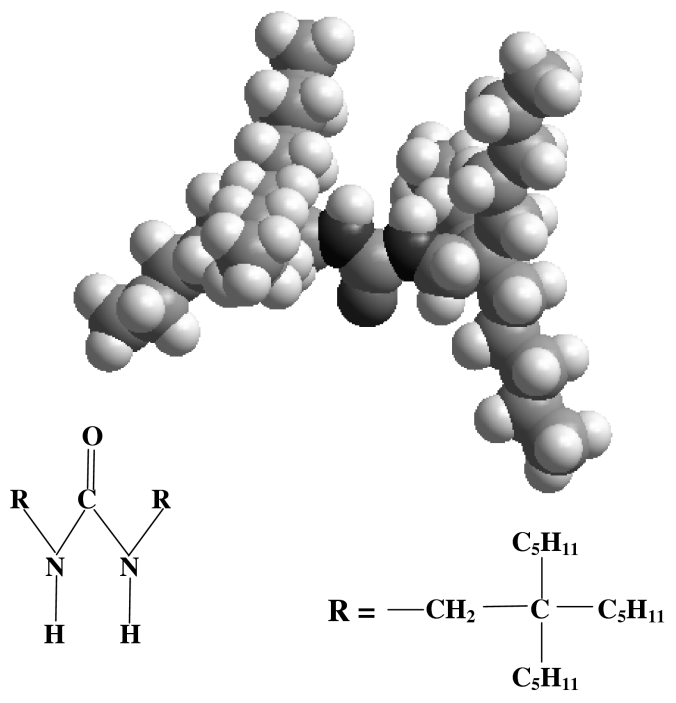

Fig. 2. $\quad N, N^{\prime}$-di(2,2-dipentylheptyl)urea (DPHU).

chloride of spectroscopic grade was purchased from Fluka. Measurements of the complex dielectric permittivity were performed in the frequency region of $100 \mathrm{kHz}$ - $100 \mathrm{MHz}$ by using a HP 4194A impedance analyzer and the measuring capacitor consisted of three plane electrodes: one central and two grounded on each side. We analyzed the dielectric relaxation spectra of $\mathrm{DPHU}+\mathrm{CCl}_{4}$ mixtures with the urea concentration up to about $7 \%$ (in mole fraction). The measurements were performed in the temperature range of $5-50^{\circ} \mathrm{C}$. 


\section{Results and discussion}

Figure 3 presents, as an example, the dielectric relaxation spectra recorded for DPHU $+\mathrm{CCl}_{4}$ solution of the urea mole fraction equal to 0.0361 , at different temperatures. In Fig. 4 the data are presented in the form of the Cole-Cole plots (imaginary part of the permittivity, $\varepsilon^{\prime \prime}$ vs. the real part, $\varepsilon^{\prime}$ ). Two features of the spectra should be noted. First of all, a remarkable strong decrease in the amplitudes of the spectra is observed when the temperature increases. It certainly results from a high temperature-sensibility of the hydrogen-bonded entities formed. Secondly, as Fig. 4 clearly shows, the deviation of the dielectric relaxation spectra from the Debye form (semi-circle in the $\left(\varepsilon^{\prime \prime}, \varepsilon^{\prime}\right)$ plane), leading to a skew form, is remarkable. That kind of anomaly in the dielectric behavior is observed for all DPHU concentrations (as presented in Fig. 5), and in the whole temperature range studied.
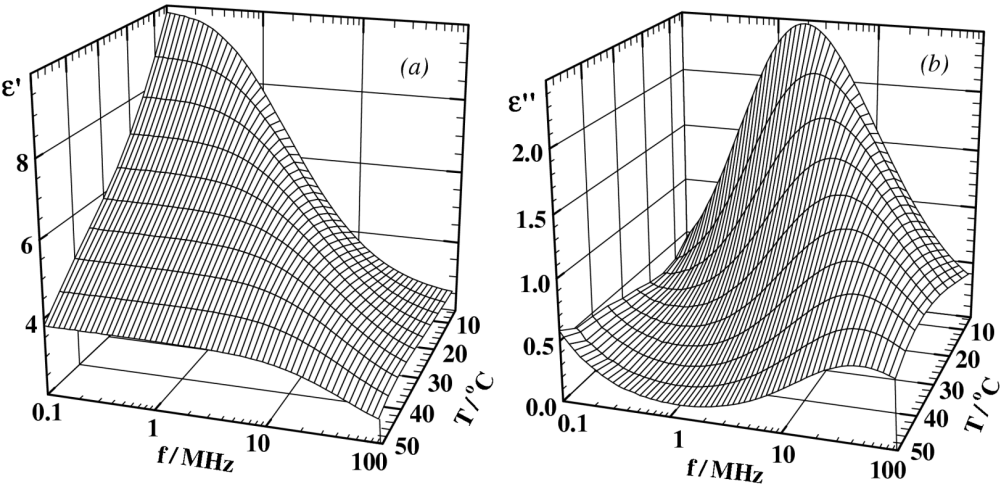

Fig. 3. Dielectric relaxation spectra (dispersion (a) and absorption (b)) recorded for $\mathrm{DPHU}+\mathrm{CCl}_{4}$ solution of the urea mole fraction $x=0.0361$.

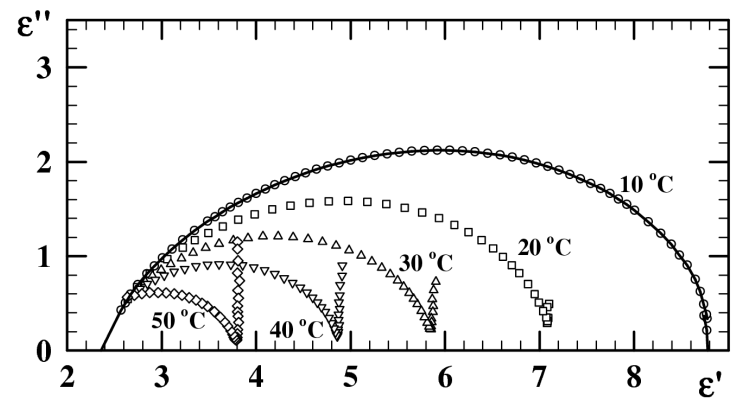

Fig. 4. The Cole-Cole plots for solution of DPHU mole fraction $x=0.0361$ in $\mathrm{CCl}_{4}$ at different temperatures. 


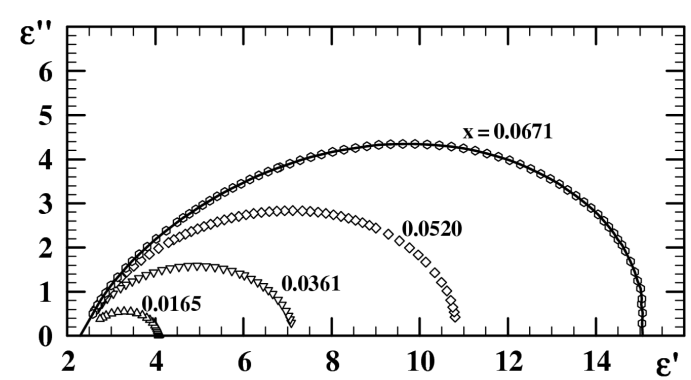

Fig. 5. The Cole-Cole plots for $\mathrm{DPHU}$ in $\mathrm{CCl}_{4}$ solutions of different urea mole fractions at $20^{\circ} \mathrm{C}$. The solid line represents the best fit of Eq. (1) to the experimental spectra.

Relaxation processes, deviating from exponential behavior in time domain or from a simple Debye form in frequency domain, are a subject of numerous works both experimental and theoretical. From the experimental point of view the problem has a long history as the dielectric relaxation anomalies occur in many physical systems such as the hydrogen bonded liquids, supercooled liquids, polymers in solutions or melts, etc. Among numerous empirical equations proposed for analysis of the dielectric relaxation spectrum departing from the Debye form, the most general one was given by Havriliak-Negami [18, 19]:

$$
\varepsilon^{*}(\omega)=\varepsilon^{\prime}(\omega)-\mathrm{i} \varepsilon^{\prime \prime}(\omega)=\varepsilon_{\infty}+\frac{\varepsilon_{\mathrm{s}}-\varepsilon_{\infty}}{\left[1+(\mathrm{i} \omega \tau)^{\alpha}\right]^{\beta}},
$$

where $\omega$ is the angular frequency of the probing electric field, $\tau$ is the relaxation time, and $\varepsilon_{\mathrm{s}}$ and $\varepsilon_{\infty}$ are the static and high-frequency limits of the real part of the complex dielectric permittivity, respectively. The fractional exponents $\alpha$ and $\beta$ reflect the broadening of the dielectric absorption band, i.e. the deviation from the Lorentzian shape (Debye model). In particular, the case of $0<\alpha<1$ and $\beta=1$ in Eq. (1) corresponds to the symmetrical broadening (the Cole-Cole equation [21]), while for $\alpha=1$ and $0<\beta<1$ one can analyze the asymmetric (skew) broadening (Davidson-Cole equation [21, 22]). When $\alpha=\beta=1$ Eq. (1) yields the Debye relaxation equation.

As a result of the best fit of Eq. (1) to the dielectric relaxation spectra of $\mathrm{DPHU}+\mathrm{CCl}_{4}$ solutions we obtained for the exponent $\alpha$ the values not too far from the unity $(0.9 \pm 0.1)$ and for the exponent $\beta$ - the value of $0.7 \pm 0.1$, in the whole range of urea concentration and temperatures studied. So, the anomaly in the dielectric relaxation behavior of the supramolecular polymer studied here is rather of the Davidson-Cole type $[21,22]$. The temperature dependences of the two remaining fitting parameters: static dielectric permittivity $\left(\varepsilon_{\text {stat }}\right)$ and the relaxation time $(\tau)$, are depicted in Figs. 6 and 7, respectively.

The conclusions arising from the data obtained seem to be important for understanding the structure and molecular dynamics of the urea-based supramolecular polymers and next — for engineering suprapolymers of a given physical property. First of all, despite rather bulky substituents R, the molecules of 


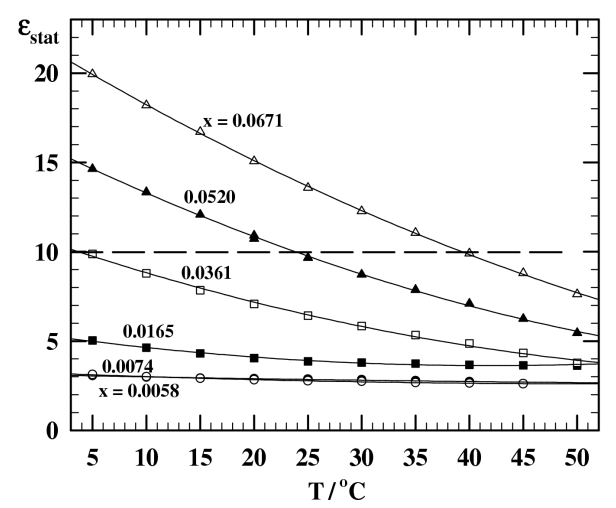

Fig. 6. Temperature dependence of the static dielectric permittivity of $\mathrm{DPHU}$ in $\mathrm{CCl}_{4}$ solutions.

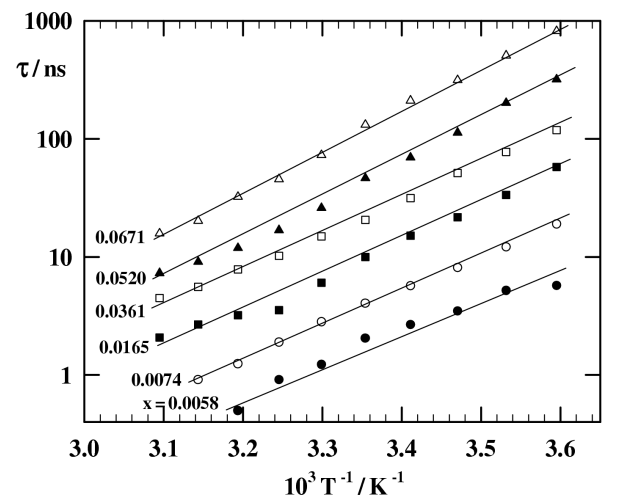

Fig. 7. Arrhenius plots of the dielectric relaxation time of DPHU dissolved in $\mathrm{CCl}_{4}$. The activation energy resulting from the slop of the dependences is equal to $60 \pm 5 \mathrm{~kJ} / \mathrm{mol}$ in the whole studied range of the urea mole fraction $x$.

DPHU show an uncommon ability to self-assembling via the hydrogen bonds $\mathrm{C}=\mathrm{O} \ldots \mathrm{H}-\mathrm{N}$, leading certainly to the formation of the linear, strongly polar entities, similar to those sketched in Fig. 1. As it is shown in Fig. 6, the static permittivity value measured for rather diluted DPHU $+\mathrm{CCl}_{4}$ solution (about $7 \%$ mole fraction) at $10^{\circ} \mathrm{C}$, has as high value (about 20) as observed in many pure polar liquids. Secondly, the Arrhenius activation energy $E_{\mathrm{A}}$, determined as a slope of the relaxation time temperature dependence (Fig. 7), is practically independent of DPHU concentration and takes a value equal to $60 \pm 5 \mathrm{~kJ} / \mathrm{mol}$, which, in terms of the hydrogen bond energy, corresponds to the breaking of three $\mathrm{C}=\mathrm{O} \ldots \mathrm{H}-\mathrm{N}$ hydrogen bonds. The value of $E_{\mathrm{A}}$ obtained is somewhat higher than that obtained for $N, N^{\prime}$-dialkylurea with small substituents $\left(\mathrm{R}=\mathrm{CH}_{3}\right.$ or $\left.\mathrm{C}_{2} \mathrm{H}_{5}\right)$ [16], where the activation energy obtained from the dielectric relaxation data roughly corresponds to the breaking of the two hydrogen bonds. 


\section{Modeling of the dielectric properties of the DPHU supramolecular solutions}

The results presented in this paper illustrate the possibilities in the creation of a supramolecular system of a given, quite precisely formulated physical property. Of course, in our case we can formulate the properties connected with the interactions polymer-electric field. The problem is not a marginal from the point of view of practical uses of supramolecular polymeric systems [1-3]. This aspect can be highlighted from two simple cases based on the dielectric results presented in this paper.

The first one concerns the static dielectric permittivity. As it is shown in Fig. 6 (dashed line), several solutions of $\mathrm{DPHU}+\mathrm{CCl}_{4}$ can take the same value of the permittivity (10, for example) at different temperatures. Figure 8 presents the real and imaginary dielectric spectra of three solutions which are characterized by $\varepsilon \approx 10$. Thus, a choice can be made between different urea concentrations and temperatures or consequently, between different frequencies of the dielectric absorption maximum $\left(f_{\max }\right)$. Figure 9 shows that, at a constant static permittiv-

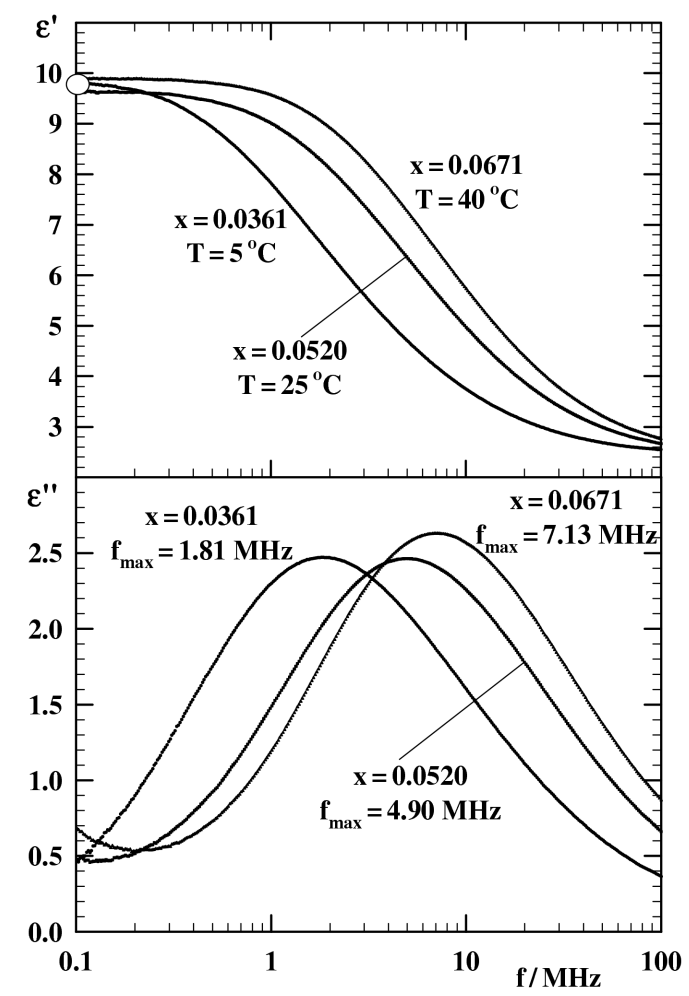

Fig. 8. The supramolecular polymer $\mathrm{DPHU}+\mathrm{CCl}_{4}$ of the static permittivity value of about 10 can be obtained in different ways, i.e. for different urea mole fractions and at different temperatures. 


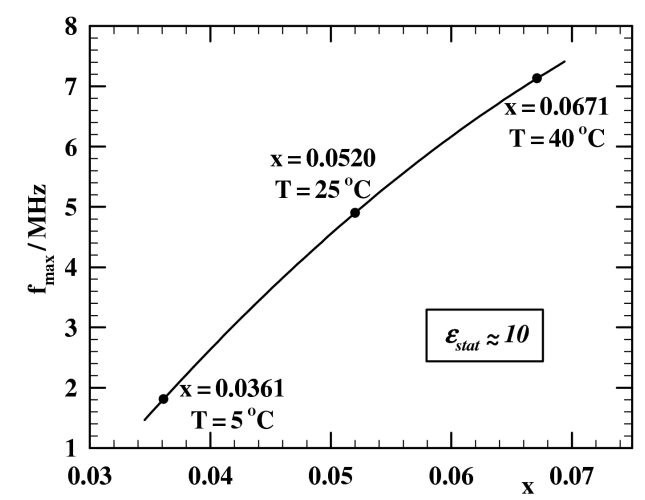

Fig. 9. For a given static permittivity value, the supramolecular polymer can be chosen between different possibilities concerning the frequency of the maximum of the dielectric absorption $\left(f_{\max }\right)$, the urea concentration, and the temperature.

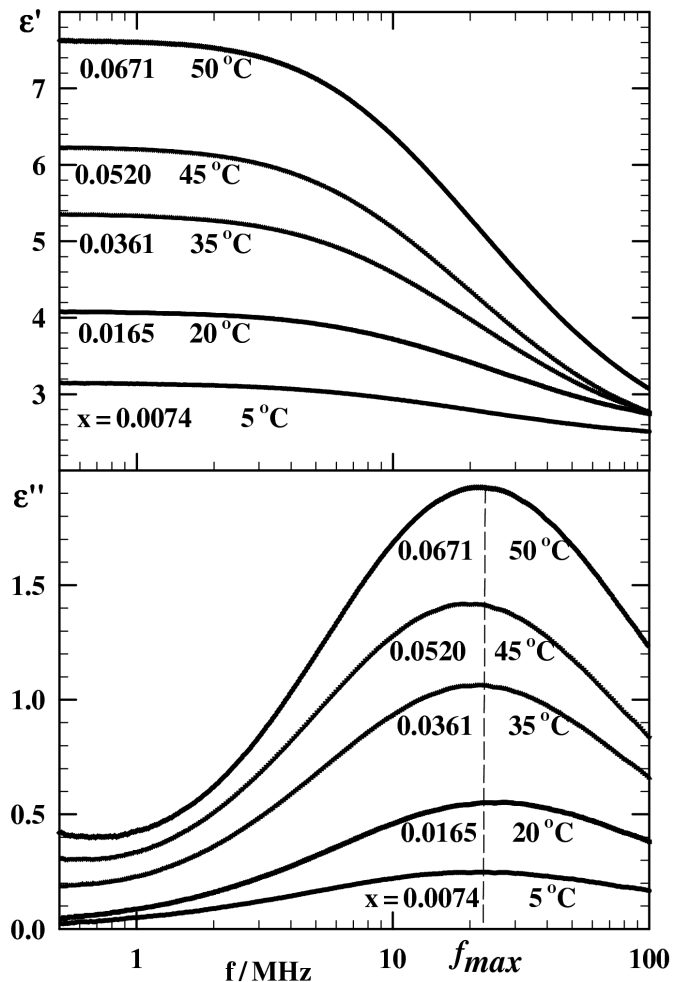

Fig. 10. The dielectric absorption maximum at the same frequency $\left(f_{\max }\right)$ can exhibit the supramolecular polymers $\mathrm{DPHU}+\mathrm{CCl}_{4}$ with different values of the static permittivity, the concentration or the temperature. 


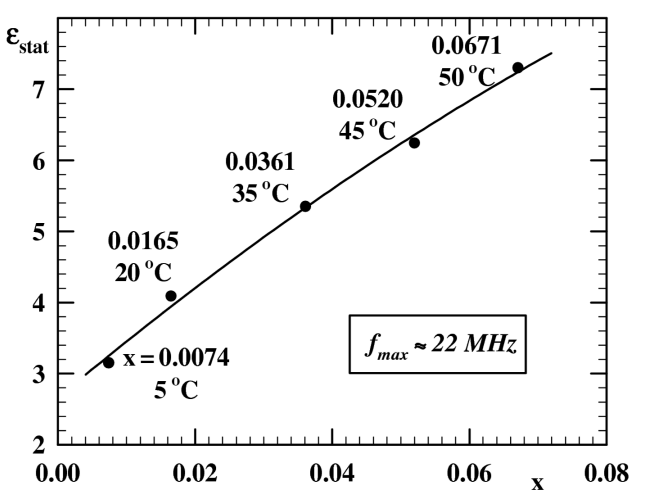

Fig. 11. Dependence of the static permittivity on DPHU mole fraction in $\mathrm{CCl}_{4}$ for a fixed frequency of the dielectric absorption maximum $\left(f_{\max }\right)$.

ity, the dependence of $f_{\max }$ on the urea concentration is quite simple, so one can precisely engineer the system in such a case.

The second possibility of monitoring supramolecular systems, concerns the solutions with the same position of the dielectric loss maximum in the frequency scale. As depicted in Fig. 10, this condition is mainly connected with a choice of the convenient value of the static permittivity of the solution. As it is shown in Fig. 11, for a given $f_{\max }$ one can engineer various solutions of $\mathrm{DPHU}+\mathrm{CCl}_{4}$ of different urea contents, at different temperatures, and consequently of different static permittivities, depending on the conditions or purpose of the supramolecular system uses.

\section{Acknowledgment}

The work was supported by the State Committee for Scientific Research $(\mathrm{KBN})$ in the framework of project No. 2P 03B 07825.

\section{References}

[1] J.-M. Lehn, Supramolecular Chemistry: Concepts and Perspectives, VCH, New York 1995.

[2] J.L. Atwood, J.E.D. Davies, D.D. MacNicol, F. Vögtle, J.-M. Lehn, Comprehensive Supramolecular Chemistry, Pergamon, Oxford 1996.

[3] A. Ciferri, Supramolecular Polymers, 2nd ed., Taylor\&Francis, Boca Raton 2005.

[4] G. Armstrong, M. Buggy, J. Mater. Sci. 40, 547 (2005).

[5] M. de Loos, J. van Esch, R.M. Kellogg, B.L. Feringa, Angew. Chem. Int. Ed. 40, 613 (2001).

[6] G. Wang, A.D. Hamilton, Chem. Eur. J. 8, 1954 (2002).

[7] J.J.E. Moreau, B.P. Pichon, M. Wong Chi Man, C. Bied, H. Pritzkow, J.-L. Bantignies, P. Dieudonné, J.-L. Sauvajol, Angew. Chem. Int. Ed. 43, 203 (2004). 
[8] M.R.J. Vos, G.E. Jardl, A.L. Pallas, M. Breurken, O.L.J. van Asselen, P.H.H. Bomans, P.E.L.G. Leclere, P.M. Frederik, R.J.M. Nolte, N.A.J.M. Sommerdijk, J. Am. Chem. Soc. 127, 16768 (2005).

[9] N. Chebotareva, P.H.H. Bomans, P.M. Frederik, N.A.J.M. Sommerdijk, R.P. Sijbesma, Chem. Commun. 39, 4967 (2005).

[10] L. Bouteiller, O. Colombani, F. Lortie, P. Terech, J. Am. Chem. Soc. 127, 8893 (2005).

[11] F. Vonau, D. Suhr, D. Aubel, L. Bouteiller, G. Reiter, L. Simon, Phys. Rev. Lett. 94, 066103 (2005).

[12] R.P. Sijbesma, F.H. Beijer, L. Brunsveld, B.J.B. Folmer, J.H.K. Ky Hirschberg, R.F.M. Lange, J.K.L. Lowe, E.W. Meijer, Science 278, 1601 (1997).

[13] L.V. Sucha, D.N. Sathyanarayana, J. Mol. Struct. 125, 89 (1984).

[14] D. Hadzi, J. Kidric, Z.V. Knezevic, B. Barlic, Spectrochim. Acta A 32, 693 (1976).

[15] M. Goodman, F. Morehouse, Organic Molecules in Action, Gordon and Breach, New York 1973.

[16] J. Jadżyn, M. Stockhausen, B. Żywucki, J. Phys. Chem. 91, 754 (1987).

[17] F. Lortie, S. Boileau, L. Bouteiller, Chem. Eur. J. 9, 3008 (1903).

[18] S. Havriliak, Jr., S. Negami, J. Polym. Sci., Part C, Polym. Symp. 14, 99 (1966).

[19] S. Havriliak, Jr., S. Negami, Polymer 8, 161 (1967).

[20] K.S. Cole, R.H. Cole, J. Chem. Phys. 9, 341 (1941).

[21] D.W. Davidson, R.H. Cole, J. Chem. Phys. 18, 1417 (1950).

[22] D.W. Davidson, R.H. Cole, J. Chem. Phys. 19, 1484 (1951). 